\title{
The imagery bizarreness effect as a function of sentence complexity and presentation time
}

\author{
SHERYL KLINE and LOWELL D. GRONINGER \\ University of Maryland Baltimore County, Baltimore, Maryland
}

\begin{abstract}
Two experiments were carried out to examine the relationship between the bizarreness effect (more words recalled from bizarre images than from common images), sentence complexity, and presentation time. Experiment 1 showed that the bizarreness effect was not influenced by processing time when simple sentences were used. However, for complex sentences, an interaction occurred such that nouns from common sentences were recalled better with an 11-sec presentation rate, and nouns from bizarre sentences were recalled better with a 15-sec presentation rate. This interaction was replicated in Experiment 2. These results show that the bizarreness effect does occur for complex sentences, provided that enough processing time is given. The results also suggest that reviews of the bizarreness literature need to consider processing time as a potentially important variable.
\end{abstract}

In 1972, Wollen, Weber, and Lowry showed that cued recall for pictures of words (e.g., piano-cigar) was no better when the words were shown in a bizarre as opposed to a common relationship. Research done since this time has also shown that the formation of bizarre images of words produces no better recall of those words when contrasted with common images, except under limited conditions. (See Einstein \& McDaniel, 1987, Einstein, McDaniel, \& Lackey, 1989, and Kroll, Schepeler, \& Angin, 1986, for reviews of this literature.) This literature suggests that the advantage of forming bizarre as opposed to common images (i.e., the bizarreness effect) occurs only for free recall as opposed to cued recall or recognition, and only when comparisons are withinsubjects as opposed to between-subjects.

The bizarreness effect was constrained further by McDaniel and Einstein (1989), who showed that the effect occurs for simple sentences (e.g., The cat chases the ball), but that the reverse effect occurs for complex sentences that have many modifiers (e.g., The yellow and orange striped cat relentlessly chases the small, scarred, rubber ball). On the surface, the results of McDaniel and Einstein seem counterintuitive. One would think that the addition of modifiers (e.g., The black and white whiskered cat chases the white fluffy cloud) would maintain or even enhance the bizarreness effect.

One possible explanation that McDaniel and Einstein (1989) give for their results is that the increased complexity of the sentences reduces the imageability of the event depicted. If this explanation is valid, it implies that the bizarreness effect for complex sentences should be sen-

The present study was based on research designed by S. Kline while enrolled in an experimental psychology course given by L. D. Groninger. Requests for reprints should be sent to Lowell D. Groninger, Department of Psychology, University of Maryland Baltimore County, Baltimore, MD 21228. sitive to different presentation times. If common images can be formed more quickly than bizarre images, then there should exist a presentation time such that a common image relationship within a sentence could be formed, but a bizarre image relationship could not be formed. A corollary of this position would be that at a certain presentation time a common image could be formed and well integrated, whereas a bizarre image could be formed but not well integrated. If these assumptions are correct, then the failure to find a bizarreness effect for complex sentences may be due to insufficient presentation time of the sentences. The present study was intended to investigate this possibility.

\section{EXPERIMENT 1}

\section{Method}

Subjects and Design. One hundred and twenty introductory psychology students at the University of Maryland Baltimore County participated for extra course credits. The design was a three-factor mixed factorial with sentence complexity (simple vs. complex) and length of processing time (short, medium, or long) as the between-subjects factors and sentence bizarreness (bizarre vs. common) as the within-subjects factor. Twenty subjects were randomly assigned to each of the six groups created by the between-subjects factor.

Materials. In order to increase the generality of words chosen as target nouns for the noun pair within each sentence, two stimulus lists of 12 sentences each were constructed for both the simple and the complex sentences. The complex sentences were adopted directly from Kroll et al. (1986). Since Kroll et al. did not use simple sentences, simple bizarre and common sentences were constructed by removing the modifiers from the corresponding complex sentences. The simple sentences used in our study averaged 7.2 words per sentence, and the complex sentences averaged 12.6 words per sentence.

Each stimulus list contained six common and six bizarre sentences. Each list also had two forms that were counterbalanced so that across subjects each sentence was presented in its common and bizarre form equally often. The common and bizarre sentences within each list were randomly ordered for each group of subjects, subject to the constraint that no more than two like sentences (two bizarre or two common) could appear consecutively. The sentences were printed on $4 \times 6$ in. index cards with the noun pair in each sentence capitalized and underlined. 
Procedure. Prior to the presentation of the stimulus list, the subjects were instructed to visualize the relationship taking place between the noun pair in each sentence. They were also asked to continue this visualization process until they were directed to rate the vividness of the sentence on a 5-point scale ( 1 indicating a clear, vivid image, and 5 indicating a poor, unclear image). Presentation and rating times varied among the six groups. Subjects viewing simple sentences were given 3,5 , or $7 \mathrm{sec}$ to visualize each sentence and $2 \mathrm{sec}$ to rate them. Subjects viewing complex sentences were given 11,15 , or $20 \mathrm{sec}$ to visualize each sentence, and $4 \mathrm{sec}$ to rate them. The subjects performed the task in groups of 3 or less, with dividers placed between them to prevent any visual contact. The cards were shown to the subjects by the experimenter, who kept each card in the subjects' visual fields for the entire length of the prescribed presentation time. Verbal signals were given to the subjects concerning when to rate the sentences and when to look at the next card.

After viewing and rating each sentence, the subjects were given a distraction task that involved choosing appropriate responses from a workbook of puzzles and then placing their answers on an answer sheet. The distraction task took $5 \mathrm{~min}$. The subjects were then allotted $4 \mathrm{~min}$ to recall as many of the underlined nouns from the stimulus list as possible.

\section{Results and Discussion}

All statistical tests were performed at the $\alpha=.05$ level. The data are shown in Table 1. A two-factor mixed analysis of variance was performed on the number of nouns recalled for both the simple and the complex sentences. There were no significant effects found for the simple sentences, but analysis of the complex sentences revealed a significant interaction between length of processing time and degree of bizarreness $\left[F(2,57)=3.17, M S_{\mathrm{e}}=\right.$ 369.71]. Subjects given $11 \mathrm{sec}$ to process each sentence recalled more common nouns, whereas subjects given 15 or $20 \mathrm{sec}$ to process each sentence recalled more bizarre nouns. Fisher post hoc $L S D$ tests revealed no significant differences between the 15 - and 20 -sec processing levels for either the common or the bizarre condition. Subjects in the complex category also recalled more bizarre than common nouns overall $\left[F(2,57)=4.57, M S_{\mathrm{e}}=369.71\right]$.

It should be noted that the recall scores were patterned in a way that was congruent with previous research. For example, the slow condition for simple sentences and the fast condition for complex sentences were similar to the conditions used by McDaniel and Einstein (1989) and yielded the same result pattern. Of specific interest are the results for complex sentences. Using an 11-sec presentation rate, McDaniel and Einstein found higher recall for common images relative to bizarre images. We too found higher recall for common images than for bizarre images, although a Fisher post hoc test showed this difference not to be significant. However, our results with complex sentences also show that when additional processing time is

\section{Table 1}

Mean Proportion of Target Nouns Recalled in Experiment 1

\begin{tabular}{lccc}
\hline \multirow{2}{*}{$\begin{array}{c}\text { Sentence } \\
\text { Complexity }\end{array}$} & $\begin{array}{c}\text { Processing } \\
\text { Time }\end{array}$ & \multicolumn{2}{c}{ Type of Imagery } \\
\cline { 3 - 4 } & $3 \mathrm{sec}$ & Bizarre & Common \\
\hline Simple & $5 \mathrm{sec}$ & .29 & .26 \\
Simple & $7 \mathrm{sec}$ & .40 & .38 \\
Simple & $11 \mathrm{sec}$ & .36 & .38 \\
Complex & $15 \mathrm{sec}$ & .52 & .37 \\
Complex & $20 \mathrm{sec}$ & .49 & .32 \\
Complex & & & .45 \\
\hline
\end{tabular}

Table 2

Mean Proportion of Target Nouns Recalled in Experiment 2

\begin{tabular}{ccc}
\hline & \multicolumn{2}{c}{ Type of Imagery } \\
\cline { 2 - 3 } Processing Time & Bizarre & Common \\
\hline $11 \mathrm{sec}$ & .29 & .43 \\
$15 \mathrm{sec}$ & .43 & .33 \\
\hline
\end{tabular}

given, the free recall advantage of bizarre images as contrasted with common images reappears.

\section{EXPERIMENT 2}

The results of Experiment 1 showed that when simple sentences are used, the bizarreness effect is not sensitive to processing time, at least over the time periods that we used. However, when complex sentences were used, an interaction occurred such that no bizarreness effect occurred at an 11-sec presentation time, but a bizarreness effect did occur at a 15 -sec presentation time. These results suggested that complex sentences do not eliminate the bizarreness effect, provided that sufficient processing time is given. Our major purpose in Experiment 2 was to check the reliability of the interaction that had occurred in Experiment 1, using complex sentences, between type of processing (common or bizarre images) and presentation times (11 or $15 \mathrm{sec})$.

\section{Method}

The design was a mixed 2 (common or bizarre images) $\times 2$ (11- or 15 -sec presentation time), with presentation time being the betweensubjects factor and type of image being the within-subjects factor. Twenty subjects were used in each between-subjects condition. The source of subjects, the complex sentence materials, and the procedures were identical to those in Experiment 1.

\section{Results}

To check on the reliability of the interaction that was obtained in Experiment 1, a $2 \times 2$ mixed analysis of variance was performed on the data shown in Table 2 . This analysis showed that the interaction between type of encoding (bizarre or common) and presentation time (11 or $15 \mathrm{sec})$ was significant $\left[F(1,22)=5.63, M S_{\mathrm{e}}=317.77\right]$, with bizarre images being recalled better at a 15 -sec presentation rate and common images being recalled better at an $11-\mathrm{sec}$ presentation rate.

\section{GENERAL DISCUSSION}

Two important findings emerge from the present study. First, contrary to the results of McDaniel and Einstein (1989), sentence complexity per se is not a limiting factor with respect to the bizarreness effect. Our results show that complex sentences need sufficient processing time before the bizarre encoding of sentences can permit free recall superior to that of common encodings. McDaniel and Einstein did increase the processing time for complex sentences relative to that for simple sentences, according to a ratio of the average number of words in complex as opposed to simple sentences. Thus relatively more time is needed to process bizarre sentences as opposed to common sentences, but the time needed is not a linear function of the number of words in a sentence.

Our results should simplify theoretical explanations of the bizarreness effect. For example, if bizarre images elicit higher recall than common images because they have a more distinctive memory representa- 
tion, then it seems that this effect ought to occur for complex as well as simple sentences. Since our results show that the bizarreness effect does occur for complex sentences, ad hoc explanations for why the effect does not occur become unnecessary.

The second important finding of the current study is that the bizarreness effect can be strongly influenced by processing time, particularly when complex materials are used. This suggests that one should examine processing time when reviewing studies pertaining to the bizarreness effect. For example, an exception to the usual finding of superior recall for bizarre sentences as opposed to common sentences is the study of Kroll et al. (1986, Experiment 1). However, in this study, complex sentences were used along with self-determined presentation times for sentences. In this study, the average presentation time per sentence (about $7 \mathrm{sec}$ ) may have been too short for the bizarreness effect to occur.

\section{REFERENCES}

Einstein, G. O., \& MCDaniel, M. A. (1987). Distinctiveness and the mnemonic benefits of bizarre imagery. In M. A. McDaniel \&
M. Pressley (Eds.), Imagery and related mnemonic processes: Theories, individual differences, and applications (pp. 78-102). New York: Springer-Verlag.

Einstein, G. O., McDaniel, M. A., \& Lackey, S. (1989). Bizarre imagery, interference, and distinctiveness. Journal of Experimental Psychology: Learning, Memory, \& Cognition, 15, 137-146.

Kroll, N. E., SChePeler, E. M., \& ANGIN, K. T. (1986). Bizarre imagery: The misremembered mnemonic. Journal of Experimental Psychology: Learning, Memory, \& Cognition, 12, 40-51.

McDaniel, M. A., \& Einstein, G. O. (1989). Sentence complexity eliminates the mnemonic advantage of bizarre imagery. Bulletin of the Psychonomic Society, 27, 117-120.

Wollen, K. A., Weber, A., \& Lowry, D. H. (1972). Bizarreness versus interaction of mental images as determinants of learning. Cognitive Psychology, 3, 518-523.

(Manuscript received June 5, 1990.) 